\title{
Effet de la fraction des fines sur le comportement d'un sable limoneux
}

\section{A. ARAB:,}

I. SHAHROUR

S. HAMOUDI ${ }^{2}$

L. LANCELOT ${ }^{1}$

'Laboratoire de mécanique de Lille (UMR 8107)

Université de Lille 1

Polytech-Lille

59655 Villenewve-d'Asco

Cedex

2 Département génie civil USTO

Université de Chlef Route de Sendjas, BP 151 02000 ChJef (Algérie)
Cet article présente une étude en laboratoire de l'influence des fines peu plastiques sur le comportement d'un sable limonesx. Létude est basée sur des essais triaxiaux drainés et non drainés qui ont été réalisés pour des fractions de fines variant entre 0 et $50 \%$. Les esseús onl été effectués sur des échantillons ayant un indice de densité $I_{f}=0,5$. Larticle est composé de deux parties. La premlère présente les sols étudiés : la seconde donne une analyse des résultats des essais réalisés et discute de l'influence des fines sur les caractêristiques mécaniques du mélange sable-limon. Les essais montrent que

l'augmentation de la teneur en fines réduit la dliatance du sol et amplifie la phase de contractance. Flle a une faible influence sur l'angle caractéristique, mais réduit l'angle de frottement du sol.

Mots-clés; contractant, drainé, fines, frottement, limon, sable, non draine, triaxial.

\section{Influence of fines fraction on the behaviour of a silty sand}

plastic fines on the behaviour of a silty and. The study s based on drained and undrained triaxial compression tests which were carried out for fractions of fines varying between 0 and 50 \% Tests were conducted on a medium dense sand with a relative density $I=0.5$. The paper is composed of two parts. The first one presents the characteristics of soils used in this study; the second provides an analysis of the experimental results and discusses the influence of fines on the mechanical characteristics of the sand-silt mixture. Tests show that the increase in the fines fraction reduces the soil dilatancy and amplifies the phase of contractance. It has a low influence on the characteristic angle. but reduces the frictional angle of the soil.

Key words : contracting drained, fines, friction angle, sand, silt, triakial. w口drained. 


\section{Introduction}

Des sols constitués d'un mélange sable-argile ou sable-limon sont frécuemment rencontrés. Les projets d'amenagement et de construction sur' ce type de sols nécessitent une bonne connaissance de leur comportement mécanique.

Des études en laboratoire ont montré que le comportement d'un mélange sable-sols fins dépend de la plasticité des sols f̂ns. Le comportement d'un nélange sable-limon dépend principalement de la teneur er. fines. En effet, jusqu'à une certaine teneur en fines. celles-ci occupent seulement le vide, et n'affectent pas d'ume manière significative le comportement du mélange. Pour cette raison, l'utilisation de l'indice des vides intergranulaire u qui considère le volume des fines comme un vide a a été suggérée pout caractérỉser ces sols (Kenny, 1977 : Mitcheli, 1993).

De nombreux travaux ont été consacrés à l'étude de la liquéfaction des mélanges sable-sols fins. Les résultats montrent qu'en fonction de la plasticité et du type des sols étudiés, l'augmentation de la fraction de fines peut conduiré à une augrantation de la résistance à la liquéfaction de ces sols (Amini et Qi, 2000), ou à une diminution de cette résistance (Shen ef al., 1997 ; Troncoso et Verdugo, 1985 ; Finn et al, 1994 ; Vaid, 1994 ; Zlatovic et Ishihara, 1997J. D'autres études ont montré que la résistance à la liçuéfaction diminue avec l'augmentation de la teneur en fines jusqu'k atteindre une résistance minimale, puis réaugmente avec la teneur en fines (Law et Ling, 1992; Koester, 1994; Buuferra et Shahrour: 2004).

Dans cet article, on présente ure étude en laboratoire du comportement d'un mélange sable-limon sur des chemins drainés et non drainés pour une tereur en fines variant entre 0 at $50 \%$. Ces essais permettent de mielix comprendre l'influence de la fraction de fines peu plastioues sur le comportement mécanjque de ce mélange. Un intérêt particulier a été porté sur l'influerice de la fraction de fines sur les variations de volume gui affectent d'une manière importante la réponse non drainée des sols, et par conséquent leur potentiel de liquéfaction. L'article est composé de deux parties. Dans lá première, on présente les matériaux utilisés, la seconde donne une analyse des résultats des essais réajisés et discute de l"influence des fines sur les caractéristiques mécaniques du mélange sable-limon.

\section{2}

\section{Matériaux étudiés}

Les essais ont été réalisés sur un mêlange du sable de Chlef (Algérie) et du limon de l'oued de Chlef. Les courbes granulométriques de ces sols sont données clans la figure 1. Le sable de Chlef est un sable moyen. avec un diametre moyen $\mathrm{d}_{50}=0,61 \mathrm{~mm}$. Le limon est peu plastique avec un indice de plasticitế de $5,8 \%$. L'étude a été réalisée pour une fraction de fines massique (Fc) allant jusquá $50 \%$. Le tableau II et la figure? donnent les variations des valeurs maximale et minimale de l'indice des vides avec la fraction de fines. On note que ces deux indices diminuent avec l'augmentation de la fraction de fines jusqu'à $F c=35 \%$ ensuite, ils réaugmentent avec la firaction de fines. Les essajs ont été réalisés à un indice de densité $I_{\mathrm{d}}=50 \%$. Les valeurs de l'ñndice des vides correspondant à cette densité sont données dans le tableau I ef la figure 2 .

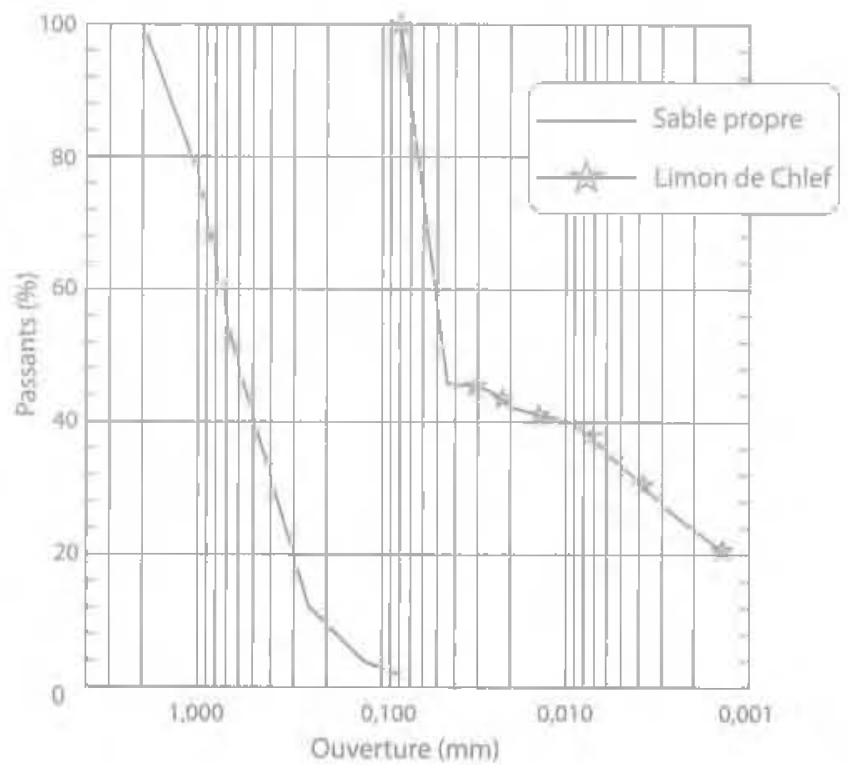

FG. 1 Courbe granulométrique des matériatux utilisés. Granular distribution for the solls used in the study.

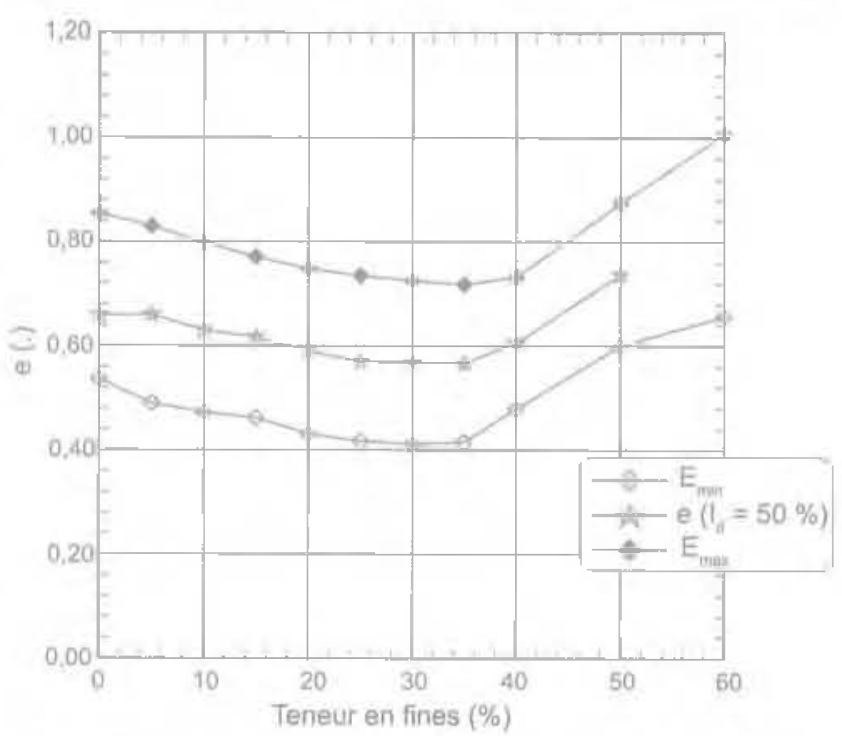

FG. 2 Variation des indices des vides maximal et miraimal avec la Iraction des fines.

Variation of the maximal and minimal void ratio of the sitty-sand mixture.

taBtzaU I Caractéristiques du mélange sable-argüle.

Gharactertstics of the sard-silt mixture.

\begin{tabular}{|c|c|c|c|c|c|c|c|c|c|c|}
\hline "\% limon & 0 & 5 & 10 & 15 & 20 & 25 & 30 & 40 & 50 & $\mathrm{EO}$ \\
\hline$a_{\operatorname{mgx}}$ & 0,854 & 0,829 & 0,798 & 0,770 & 0,748 & 0.735 & 0,718 & 0,732 & 0,874 & 1,007 \\
\hline $\mathrm{E}_{\text {тия }}$ & 0,535 & 0,49 & 0,472 & 0,462 & 0.431 & 0.417 & 0.412 & 0,478 & 0,600 & 0,657 \\
\hline e $\left.: \|_{t \mid}=50 \%\right\}$ & 0,859 & 0,659 & 0.630 & 0,616 & 0.589 & 0,570 & 0.568 & 0,605 & 0,605 & - \\
\hline
\end{tabular}


Selon différents auteurs (Kenny, 1977 ; Mitcheli, 1993), Le comportement d'un mélange sable-limon dépend de l'jndice des vides tntergranulaire $\left\{e_{5}\right.$ )

$$
\mathrm{e}_{5}=\frac{V_{v}+V_{4}}{V_{8}}
$$

$\mathrm{V}$. $\mathrm{V}$, et $\mathrm{V}$ désignent les volumes de vide, des fines et des grains solides, respectivement.

Quand les masses spécifiques du limon et du sabje sont très proches, cet indice (e) peut être déterminé en fonction de lindice des vides global (e) et de la fraction des fines (Fcl par l"expression suvante (Thevanayagam, 1998) :

$$
e_{s}=\frac{e+(F c / 100)}{1-(F c / 100)}
$$

La figure 3 montre la variation de l'indice des vides intergranulaire avec la teneur en fines (Fc) pour l'indice de densité $I_{d}=50 \%$. On note que cet indice augmente de 0.7 à 2.4 quand la fraction des fines crolt de 0 ă $50 \%$.

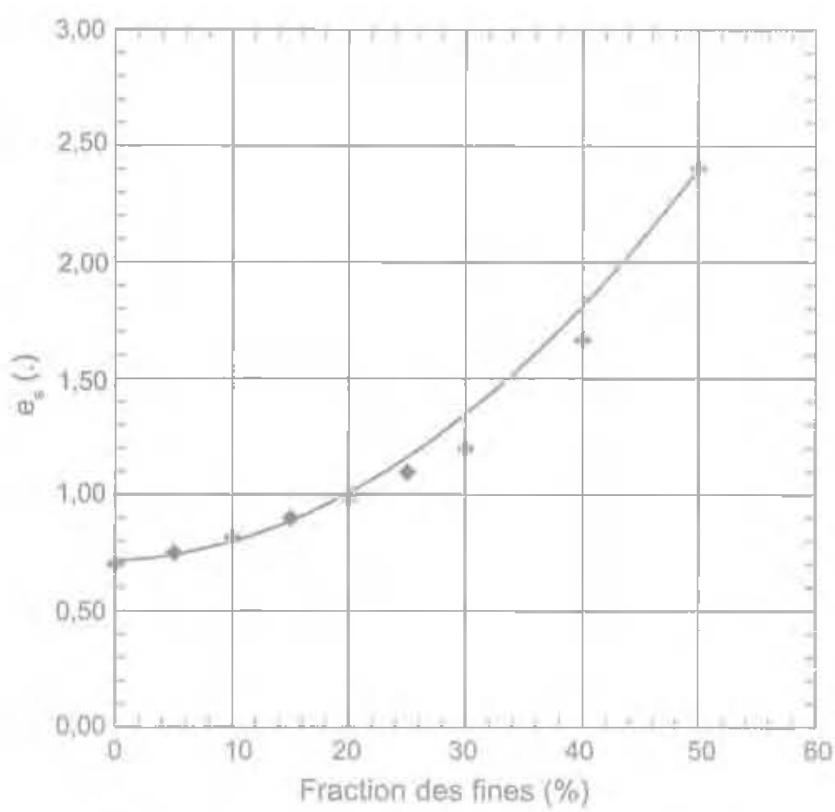

FIG. 3 Variation de l'indice des vides intergranulaire en fonction de la teneur" en fines.

Vartiation of the inter granular woid ratio with the fraction of tines.

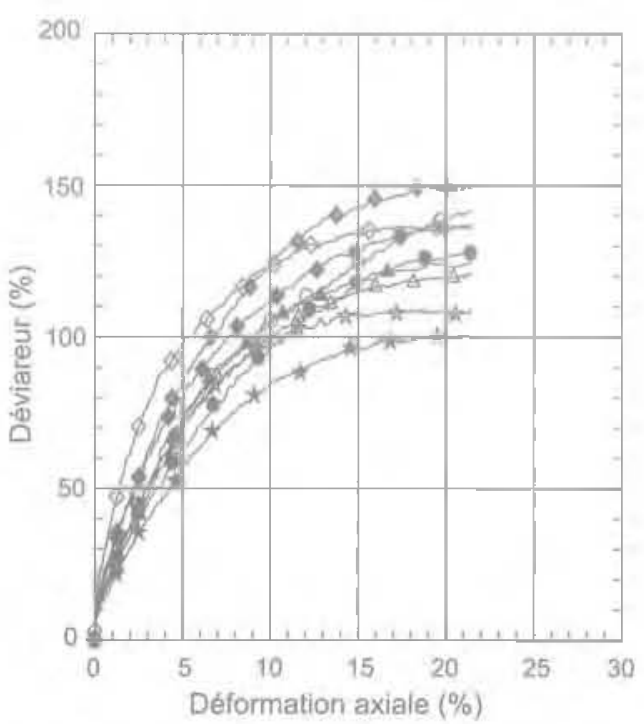

\section{Résultats des essais réalisés}

\section{4}

\section{Essais de compression drainée}

La figure 4 montre les résultats des essais de compression drainée réalisés à une pression de confinement de $100 \mathrm{kPa}$ pour des fractions de lines comprìses entré zéro (sable propre) et $50 \%$. On note que la fraction des fines affecte d'une manière sensible les variations du déviateur et de la déformation volumigue. I"augmentation de la fraction de fines entre 0 e $50 \%$ induit une diminution de la raideur initiale du sol et de la résistance du sol (déviateur maximal). Err ce qui concerne la ctéformation volumique, on note que le sable propre et les échantillons a fatble fraction de fines $(\mathrm{Fc}<20 \%$ ) présentent une phase de contractance suive d'une phase de dilatance. Pour le sable propre et l'échantillon $5 \%$ de fines, la phase cle dilatance apparaît à partir de $8 \%$ de déformation axiale, tandis que pour les échantillons ayant une teneur en fines de 10 à $30 \%$ la dilatance est retardée et apparât à partir dure déformation axiale de $13 \%$. Pour les échantillons avec une teneur en fines de 40 et $50 \%$, on observe uniquement une phase de contractance. La figure 5 montre les courbes de variation des déformations volumiques à l'état' caractéristique (changement de phase contractance-dilatancel et à la plasticité parfaite en fonction de la teneur en fines. On remarque que l'écart entre ces courbes décroit avec l'augmentation de la fraction des Fines, traduisant la disparition de la phase de dilatance él'apparition uniquement de la phase de contractance après $30 \%$ de fraction de fines.

\section{2}

\section{Essai de compression non drainée}

La figure 6 montre les résultats des essais non drainés réalisés pour différentes valeurs de la traction de fines (entre 0 et $50 \%$ ) à une pression de confinement initial de $100 \mathrm{kPa}$. Tous les essais ont été réalisés aved une contre-pression de $500 \mathrm{kPa}$. On note que l'aug-
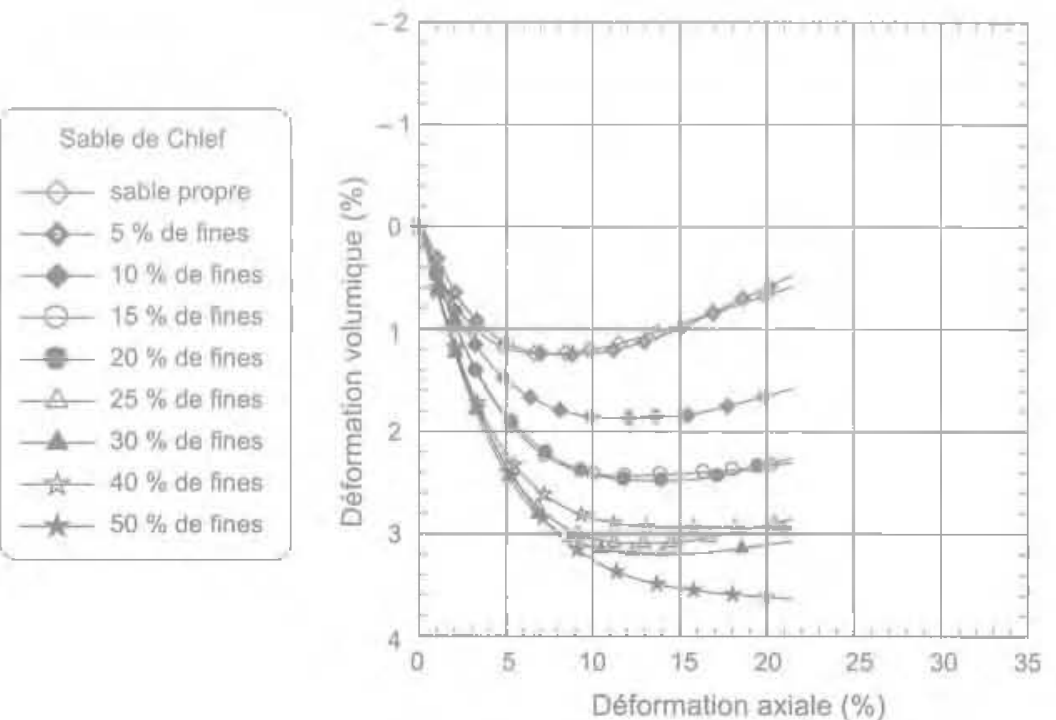

ตa. Influence de la teneur en fines sur la réponse crainée du mélange sable-limon.

Influence of the content of fines on the drained response of the sand-silt mixture. 


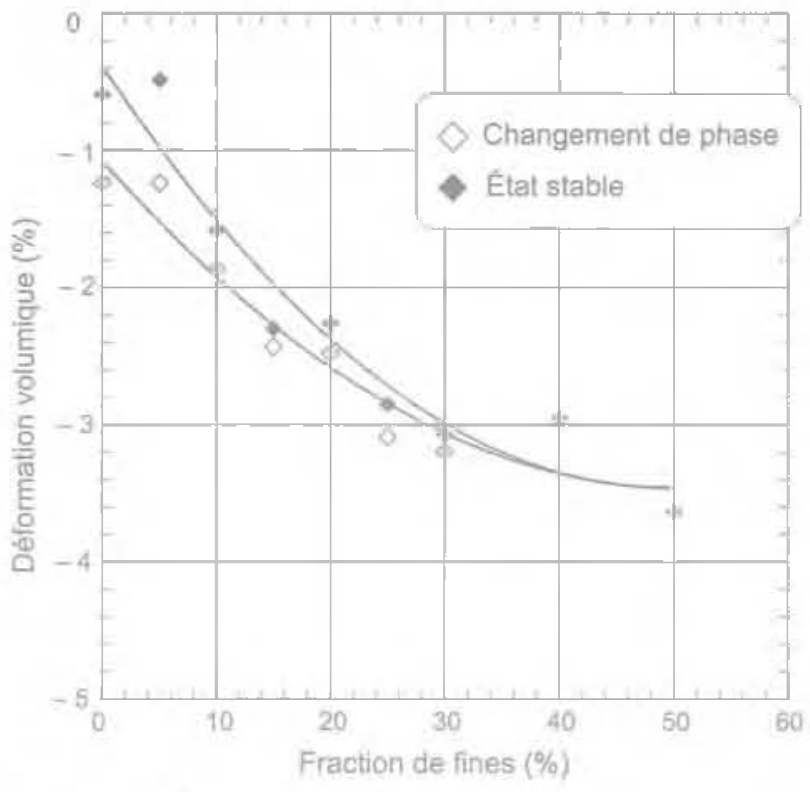

Ma.s Influence de la fraction de fines sur les défomations volumicues au changement de phase et à la stabilisation.

Influence of the fines fraction on the wolume deformations at the phase change and at stablization:

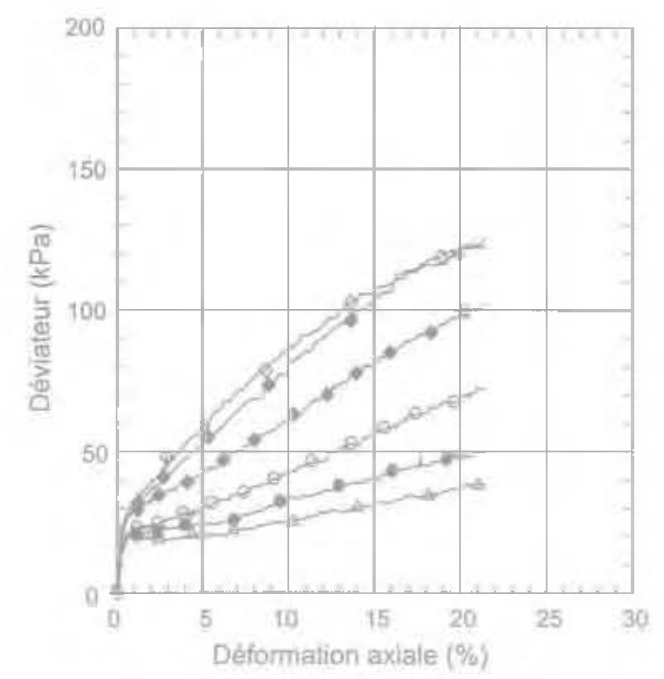

mentation de la fraction de fines conduit à une augmentation de la pression interstitielle. Cette augmentation résulte du rôle des fines dans laugmentation de la contractance du mélange observée lor's des essais dranés. L'augmentation de la pression interstitielle conduit à une réduction de la contrainte effective de confinement et par conséquent à une réduction de la résistance du mélange au déviateur comme l'illustre la figure 6a. Le chemin de contrainte dans le plan ( $\mathrm{p}^{\prime}, \mathrm{q}$ ) montre bien le rôle de l'augmentation des fines dans la réduction de la pression moyenne effective.

La figure 7 montre les résultats des essais de compression non drainée réalisés à un confinement initial de $20 \mathrm{kP}$. Ces résultats sont qualitativement idertiques à ceux obtenus pour la pression de confinement de $100 \mathrm{kPa}$ avec sur le plan quantitatif une amplification de l'effet de la fraction de fines sur la variation de la pression interstitielle et de la résistance du mélange sous chargement déviatorique. Dans ce cas, l'influence des fines sur le comportement non drainé du melange est observée pour les faibles tereurs en fines (5 et $10 \%$ ), et devient très prononcée au-celă de $15 \%$. Ces résultats sont en accord avec les observations de Shen et al. (1977) et de Troncosco et Verdugo (1985).

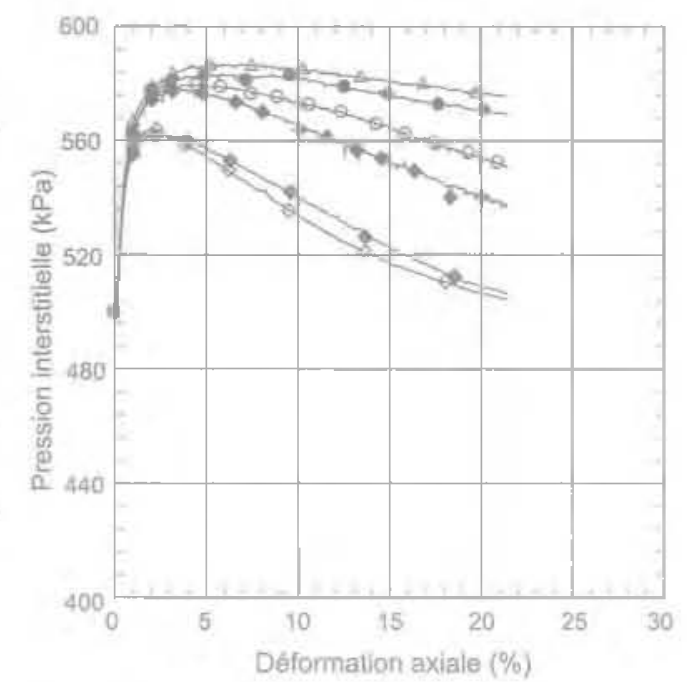

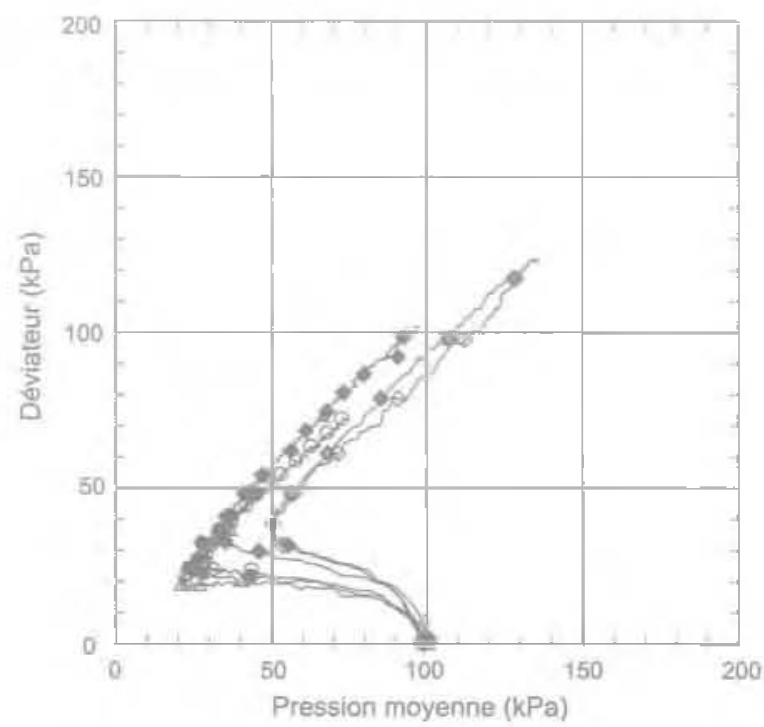

FG. Influence de la teneur des fines sur le comportement non diainé d'un mélange sable-limon (confinement initial = $100 \mathrm{kPa}$. Influence of the fines fraction on the undrained belhaviour of the silty-sand mixture (jonitial effective lateral stress = $100 \mathrm{kPal}$.

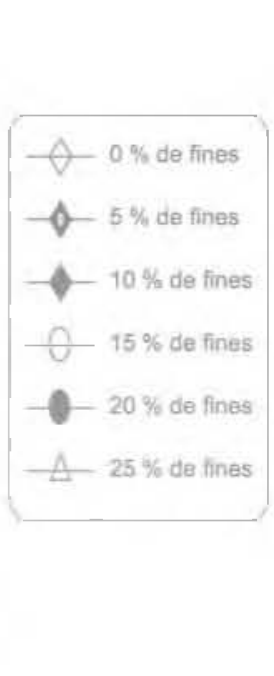



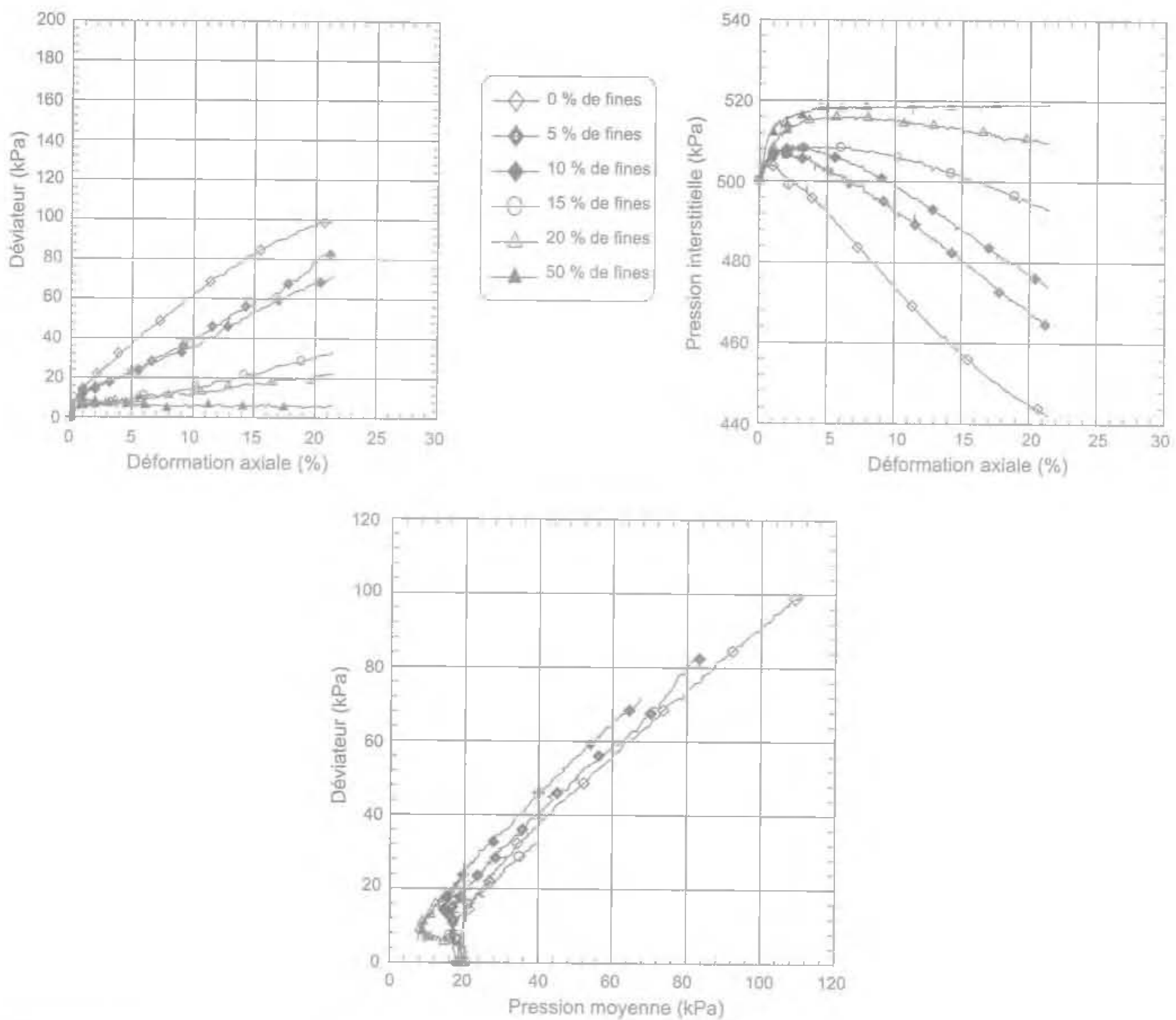

Fa. 7 lлfluence de la teneur des fines sur le comportement non draỉné d'un mélange sable-limon [confinement initial $=20 \mathrm{kPa}$ ).

Infiuence of the fines Fraction cr the undrained behaviour of the silty-sand mixture (initual effective lateral stess = $20 \mathrm{kPa}$,

\section{Influence des fines sur les caractéristiques mécaniques}

La figure 8 montre l'évolution du module de déformation sécant $\left(\mathrm{q} / \varepsilon_{\mathrm{s}}\right)$ en fonction de la défomation axiale $\left[\varepsilon_{0}\right]$. On observe que ce module décroit avec l'augmentation de la teneur en fines. Cette diminution est très significative jusqu"à une déformation de $0,7 \%$, ensujte le module sécant tend à se stabiliser. La fïgure $8 \mathrm{~b}$ montre linfluence de la fraction de fines sur le module de déformation sécant mesuré a $\mathrm{E}_{\mathrm{a}}=1 \%$. On note que ce module décrolt avec l'augmentation de la fraction de fines jusqu'â $\mathrm{FC}=20 \%$, ensuite il terd à se stabiliser.
La figure Ga montre l’influence de la fraction de fines sur l'angle de frottement du mélange sable-limon. On note que cet angle décroît (selon une relation quasi linéaire) de 24 a 20 degrés quand la fraction des fine croit de 0 à $50 \%$.

La figure 9b montre la variation de l'angle caractéristique (changement de phase contractance/dilatance) en fonction de la teneur en fünes. On remarque que la teneur en fines n'a pas d'influence sur l'angle caractéristique : la valeur de cet angle est d'environ 21 degrés pour les mélanges étudiés. La réduction de l’angle de frottement avec l'augmentation de la fraction de fines et la faible influence de cette demière sur l'angle caractéristique expliquent le rôle de la fraction de fines dans l'augmentation de la phase de contractance des sols étudiés. 


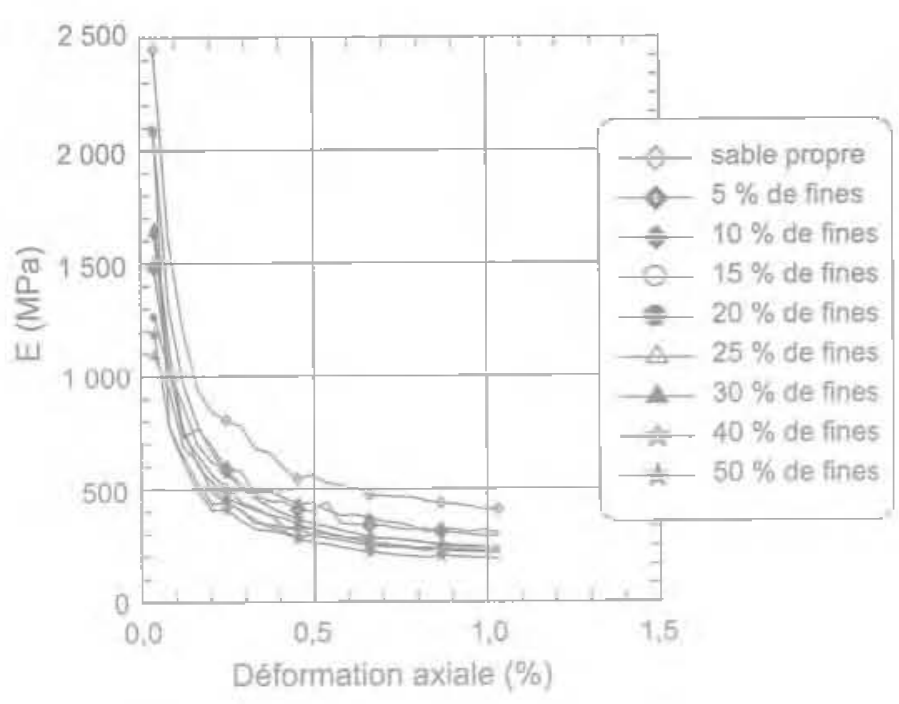

(a)

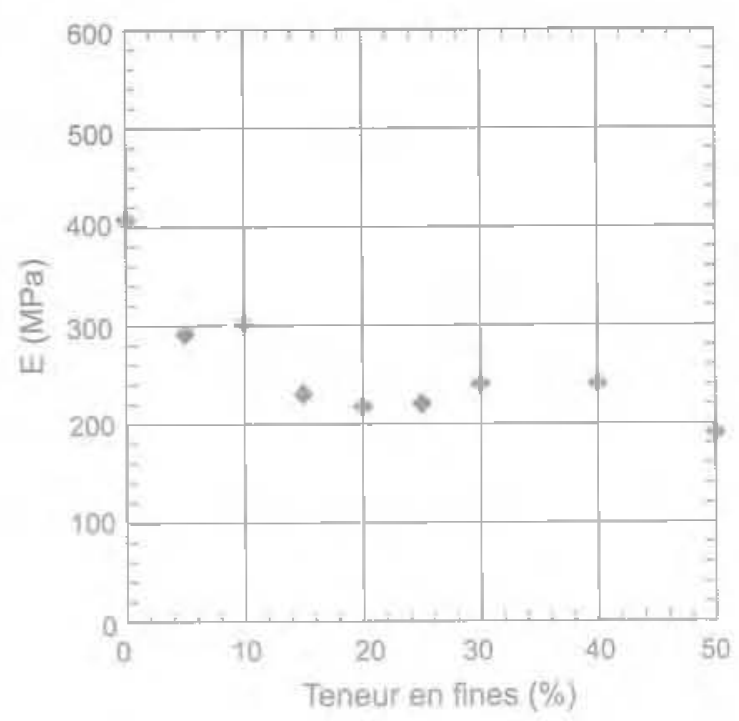

(b)

F1ร. Infuence de la fraction de fines sur le motiule de déformation sécant. a) Variation du module sécant avec la dëformation axiale; b) module sécant à $\mathrm{E}_{\mathrm{a}}=0,01$, Influerce of the fines fraction on the secant modulus.

a) Variation of the secant modulus with the axial strain ; b) secant modulus at $\mathrm{E}_{\mathrm{a}}=0.01$.



(a)

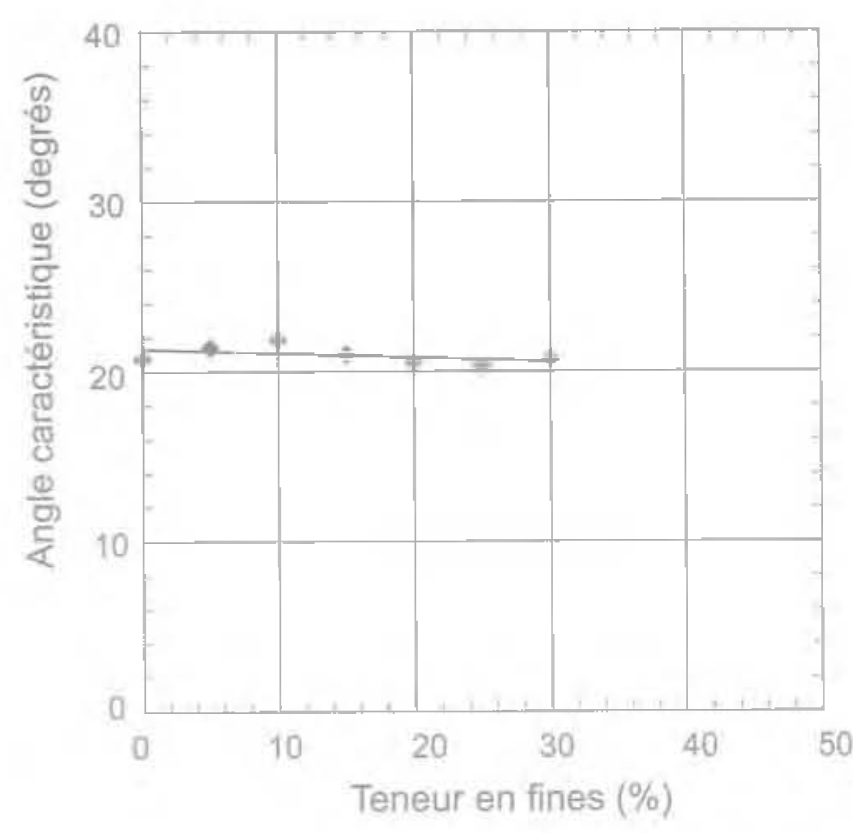

(b)

Infuence de la fraction des fines sur l'angle de frottement et l'angle caractéristique. influence of the fines fraction on the frictional and characteristic angles.

Les essais montrent que l'augmentation de la teneur en fines induit une réaction de l'angle de frottement du sol-limoneux (l'angle de frottement décroit de 24 degrés à 20 degrés quand la fraction de fines croït de 0 à $50 \%$ ). mais affecte peu l’angle caractéristique. Ceci se traduit par un effet important sur le comportement volumique qui se manifeste par une amplification de la phase de contractance lorsque la fraction de fines augmente. Cet effet est également important pour le comportement des sables limoneux sur des chemins non drainés à cause du fort couplage entre le comportement déviatorique et volumique pour ce type de chemins. 


\section{Bibliographie}

Amini F. OA G.Z. - Liquefaction testing of layered silty sands. Journal of Geotech. nical Engineering. ASCE, vol. $126, n^{\circ} 3$. 2000, p. 208-217.

Bouferra R. Shahrour 1. - Influence of fines on the resistance to liquefaction of a clayey sand. Ground Improvemen! 8 , $n^{\circ}$ 1. 2004. p. 1-5.

Finn W.L. Ledbetter R.H., Wu G. - Liquefaction on silty soils : Design and analysis. Ground failures under seismic condifion. Geotechnical special pubijcation 17 ${ }^{\circ} 44$. ASCE, 1994, 0. 51-76.

Kenny LC. - Residuel strength of minera mixtures. Proc. 9th Lnt. Conf. Soil Mech. and Found. Eng. Tokyo, vol. 1, 1977, p. 155-160.
Koester J.P. - The influence of fine type and content on cyclic strength. Ground failures under selisnic condition. Geotechnical special publication no 44, ASCE, 1994, p. 17-33.

Law K.T. Ling YH - Liquefaction of granular soils with non-cohesive and cohesive fines. Proc, of the 10th World Conferance on Earthquake Engineering. Potterclam, 1992, p. 1491-1496.

Mitchell J,K. - Fundamental of soil behaviour. John Wiley Interscience. New York, 1993, 2nd ed

Shen C.K. Vymoed J.L., Uyeno C.K. - The effects of fines on liquefaction of serids. Proc. Oth Int. Conf. Soll Mech. and Found. Eng, Tokyo, wol. 2, 1977, p. 381-385.
Thevarayagam S. - Effect of fines and conffring stress on undrained shear strength of silty sands. I. Geotech. Geoenviron. Eng DW. ASCE, 124, $\pi^{\circ}$ 6, 1998, p. $479-491$

Ironcosco . IH., Verdugo R. - Silt content and dyranic behaviour of tailing sands. Proc 12th Int. Conf. on Soil Mech. and Found Eng. San Francisco, 1985, p. 1311-1314.

Vaid V.P - Liquefaction of silty soils. Ground failures under seisnic condition. Geotechrical Special publication $n^{\circ} 4.4$ ASCE, 1994, D. 11-16.

Zlatovic S., Tshihara K. - Normalized behavjour of very loose non-plestic soils Effects of fabric. Soils and Foundations. vol. $37, r^{\circ} 4,1997,0.47-56$. 